\begin{tabular}{|l|l|l|l|}
\hline Eiszeitalter u. Gegenwart & 38 & $128-129$ & Hannover 1988 \\
\hline
\end{tabular}

\title{
Über einen zweiten Urmenschen-Fund aus dem Neandertal
}

\author{
MANFred SCHÜRmANN *)
}

Hominidae, Neandertal, cave, fossil locality, skeleton, skull, Löbbecke-Museum

North Rhine-Westphalia

Kurzfassung: 1895 wurden im Neandertal Reste eines zweiten Urmenschen-Skelettes gefunden. Diese gingen ohne wissenschaftliche Bearbeitung in die Literatur als Homo neanderthalensis II ein. Die Knochenreste gelten heute als verschollen.

\section{[On the Discovery of a Second Primitive Man in the Neandertal]}

\begin{abstract}
In 1895 parts of a second skeleton of a primitive man were found in the Neandertal. This fact entered into literature as Homo neanderthalensis II, without any scientific description. Nothing ever has been heard of these bones since.
\end{abstract}

[Au sujet de la découverte d'un second homme primitif dans le Neandertal]

Résumé: Les restes d'un second squelette d'homme primitif out étè découverts en 1895 dans le Neandertal. Ils furent répertoriés dans la littérature sous le nom d'Homo neanderthalensis II mais sans investigation scientifique rigoureuse et out disperus depuis.

Beim Ausräumen einer kleinen Höhle im Neandertal bei Düsseldorf, der Feldhofer Grotte, stießen im Sommer 1856 zwei Arbeiter auf Knochenreste eines menschlichen Skelettes. Die Knochenreste lagen in Längsrichtung der Höhle, der Kopf zum Höhlenausgang hin, in $60 \mathrm{~cm}$ Tiefe im Höhlenlehm. Beim Fortschreiten der Arbeit stießen die Steinbrucharbeiter natürlich zuerst auf den Schädel. Durch den anhaftenden Höhlenlehm erkannten sie ihn nicht und beförderten ihn mit dem übrigen Höhlensediment auf Halde. Eine spätere Nachsuche förderte lediglich die Kalotte als Teil des Schädels zutage (FUHLROTT 1859: 136-137). FUHLROTT erkannte seinerzeit die Bedeu-

*) Anschrift des Autors: M. SCHÜRMANN, Iserlohner Str. 32, D - 4000 Düsseldorf. tung des Fundes, der als klassischer Neandertaler Eingang in die Literatur fand. SCHAAFFHAUSEN (1888: 8), der als erster den gesamten Fund aus dem Neandertal abbildete, äußerte gleich die Vermutung, es könne sich hierbei wohl um eine Bestattung handeln.

In den nachfolgenden Jahrzehnten sind zwar bei der weiteren Ausdehnung des Steinbruchbetriebes Knochenreste der pleistozänen Tierwelt gefunden worden, eine systematische Untersuchung der Lehmausfüllungen der Höhlen auf tierische bzw. menschliche Knochenreste oder Artefakte war unterblieben. Das aufgesammelte Knochenmaterial ging in die Sammlung des Naturwissenschaftlichen Vereins zu Düsseldorf über (JANSEN 1892: 36-37).

Auf der 70. Versammlung der Gesellschaft Deutscher Naturforscher und Ärzte, Düsseldorf, September 1898, stellte der Archäologe RAUTERT (1899) einen weiteren, 1895 getätigten Fund menschlicher Knochen aus dem Neandertal vor. Die Knochenreste lagen ausgestreckt in der Längsrichtung einer kleinen Höhle, die durch den Steinbruchbetrieb bereits bis zur Rückwand abgebrochen war. Der Fund, der in einer Tiefe von ca. $50 \mathrm{~cm}$ im Höhlenlehm lag, bestand aus folgenden Knochen:

2 Femura

2 Tibia

2 Humeri

1 Beckenfragment (rechte Seite)

1 Kniescheibe

mehrere Rippenstücke

Die hier aufgeführten Knochen deuten auf ein ehemals vollständiges Skelett hin. Es dürfte sich hierbei, ebenso wie beim klassischen Neandertaler, um eine Grablage handeln. Ein Schädel wurde nicht entdeckt, er lag vermutlich zum Höhleneingang gerichtet und wurde beim Ausräumen des Höhlenlehmes übersehen, im Gegensatz zu den langgestreckten Arm- und Beinknochen. Viele Parallelen bestehen also zum 
FUHLROTT'schen Fund von 1856. Die Fundstelle selbst lag etwa $180 \mathrm{~m}$ nordöstlich der Feldhofer Grotte (KOENEN 1904a: A 36).

Der Archäologe KoENEN, der erste systematische Ausgräber eines römischen Kastells in Deutschland (SEELING 1984), mit RAUTERT freundschaftlich verbunden, nahm sich in der Form des Fundes an, daß er einen wissenschaftlichen Streit über die stratigraphische Lagerung der Höhlenlehme im Neandertal vom Zaune brach, vor allem mit dem Geologen RAUFF (KOENEN 1904b). KOENEN blieb aber unbelehrbar, er wollte unbedingt den klassischen Neandertaler im Tertiär ansiedeln. Der RAUTERT'sche Fund bekam in seinen Streitschriften voreilig die Bezeichnung „Homo neanderthalensis II". Leider ist der Fund nie wissenschaftlich bearbeitet worden, lediglich fand durch den Anatomen KNAATSCH eine oberflächliche Beurteilung statt (KOENEN 1902: A 72; RAUFF 1904: 74).

Nach dem Tode von RaUTERT, 1902, ging seine Sammlung in den Besitz des Naturwissenschaftlichen Vereins zu Düsseldorf über. „Die Knochenreste aus dem Neandertal, die namentlich durch Herrn RAUTERT dem Verein gesichert wurden, sind ebenfalls jetzt, nachdem der nötige Raum zur Verfügung stand, wenigstens zugänglich gemacht, und harren der Bearbeitung durch die Hand eines Spezialmannes; sie sind gruppiert nach ihrer Zusammengehörigkeit bezüglich der mutmaßlichen Lagerstätte" (HÜLSKÖTTER 1909). Die Sammlungen des Naturwissenschaftlichen Vereins gingen gemäß den Statuten in den Besitz der Stadt Düsseldorf über und wurden in den Jahren 1914 bis 1919 in den Bestand des heute noch bestehenden Löbbecke-Museums eingegliedert (STADTARCHIV DüSSELDORF). Das Löbbecke-Museum selbst wurde 1904 als Stiftung THEODOR LÖBBECKES der Stadt Düsseldorf übergeben, um seiner berühmten Konchyliensammlung einen würdigen Platz zu verleihen (WENK 1911).

Vermutlich sind die Knochenreste aus dem Neandertal durch Kriegseinwirkung vernichtet worden. In den Beständen des Löbbecke-Museums ist dieser Fund nicht mehr vorhanden. So ist der vermutlich zweite Fund eines Urmenschen aus dem Neandertal für immer verschollen.

\section{Schriftenverzeichnis}

FunLrotT, C. (1859): Menschliche Überreste aus einer Felsengrotte des Düsselthals. - Verh. Naturhist. Ver. preuß. Rheinl. u. Westf., 16: 131-153, Taf. 1; Bonn. - [Reprint 1986, Zweckverband Erholungsgebiet Neandertal].

HÜLSKötTeR, C. (1909): Bericht über die Sammlungen. Mitt. Naturw. Ver. Düsseldorf, 4: 83-84; Düsseldorf.

JANSEN, K. (1892): Vermehrung der geognostischen und geologischen Sammlung. - Mitt. Naturw. Ver. Düsseldorf, 2: 33-37; Düsseldorf.

KoEnEn, C. (1902): Zur Altersbestimmung der Neanderthaler-Menschenknochenfunde und anderer Höhleneinschlüsse des Devonkalksteinzuges. - Niederrh. Ges. Natur- u. Heilkde.: A 64-A 77; Bonn.

- (1904a): Eigenart und Zeitfolge des Knochengerüstes der Urmenschen. - Niederrh. Ges. Natur- u. Heilkde.: A 19-A 39; Bonn.

- (1904b): Die Zeitstellung der beiden Neandertalmenschen. - Niederrh. Ges. Natur- u. Heilkde.: A 63 -A 73; Bonn.

RauFF, H. (1904): Über die Altersbestimmung des Neandertaler Menschen und die geologischen Grundlagen dafür. - Verh. Naturhist. Ver. preuß. Rheinl. u. Westf., 60: 11-90, Taf. 1; Bonn.

RAuTERT, O. (1899): Über Funde aus dem Neanderthal. - Verh. Ges. dt. Naturf. u. Ärzte, 2. Tl., 1. Hälfte, Naturwiss. Abt: 188-190; Leipzig.

Schaaffhausen, H. (1888): Der Neanderthaler Fund. 49 S., 3 Taf.; Bonn.

SeEling, H. (1984): Constantin Koenen (1854-1929). 137 S., 30 Abb.; Neuss.

STADTARCHIV DúsSELdoRF: Verwaltungsbericht der Stadt Düsseldorf für den Zeitraum vom 1. April 1914 bis 31. März 1919: 171-173.

WENK, W. (1911): Das Löbbecke-Museum. - Mitt. Naturwiss. Ver. Düsseldorf, 5: 135-139; Düsseldorf.

Manuskript eingegangen am 17. 3. 1987. 